\title{
Ich höre ... nicht alles!
}

\section{Partizipation und Interaktion von Lernenden im Fokus Hören}

DOI: https://doi.org/10.53349/sv.2021.i3.a120

\begin{abstract}
Kinder und Jugendliche mit Gehörlosigkeit oder mittel- bis hochgradigen Hörbeeinträchtigungen sind im schulischen Alltag oftmals mit, für Lehrpersonen nicht sichtbaren, Barrieren ausgesetzt, die sie beim Lernen, Interagieren und Partizipieren behindern. Wenn auch vorab bauliche und strukturelle Rahmenbedingungen mitbedacht werden, so prägen rückblickend Erfahrungen der sozialen Ausgrenzung und Misserfolg die Schullaufbahn von Menschen im Fokus Hören. Dieser Beitrag möchte allen Personen, die mit Kindern und Jugendlichen im Fokus Hören leben und arbeiten, Hoffnung geben und ebenso Wege skizzieren, wie der Abbau von Barrieren und die Bereitstellung von passenden Strukturen und Ressourcen gelingen kann, sodass diese Lernenden in Schule und Unterricht partizipieren, innerhalb der Peergroup angepasst interagieren und autonom, ihren Bedürfnissen entsprechend, handeln können.
\end{abstract}

Partizipation, Hören, Beeinträchtigung, Hoffnung

Alle Lehrer [sic] sollen genau hinschauen, was ich zum Lernen brauche. Dann wissen sie auch, dass ich mich nicht so lange konzentrieren kann ..., dass ich sie nicht verstehen kann, wenn sie zur Tafel sprechen und ich ihre Lippen nicht lesen kann ...

Daniel $^{1}, 12$ Jahre

Mein Besuch an einer Mittelschule in Niederösterreich und das Gespräch mit einem Schüler vor Ort boten mir einen Einblick in die Lern- und Arbeitsweisen sowie -räume von Kindern und Jugendlichen im Fokus Hören. Es macht Mut und Hoffnung und es bewegt, Kindern und Jugendlichen zuzuhören, sie wahr- und in ihren Wünschen, Sorgen und Ängsten erstzunehmen. 
Daniel besucht die 5. Schulstufe. Schon im Kleinkindalter wurde er beidseitig mit einem $\mathrm{Cl}$ (Cochlea-Implantat) versorgt und hat gelernt zu hören. Doch dieses Hören ist nicht mit dem Hören eines hörenden Menschen zu vergleichen. (Leonhardt, 2011) Immer wieder gibt es Situationen, in welchen Daniel der akustischen Reizüberflutung nur entfliehen kann, indem er seine Cls deaktiviert, oder Zeitpunkte, innerhalb welcher er ganz genau auf die Lippen schauen muss, um Wichtiges aus Unwichtigem zu filtern. Diesen herausfordernden Lebens- und Lernlagen begegnet der Jugendliche oftmals mit Rückzug und fühlt sich in diesen Momenten von seiner Umwelt, den Schulkolleg*innen und den Lehrpersonen weder verstanden, noch akzeptiert. Es fällt inm schwer, Freundschaften zu schließen und diese aufrecht zu erhalten. Er erlebt sich selbst als Außenseiter und vor allem in den Unterrichtsgegenständen mit sprachlichem Schwerpunkt, wie Deutsch und Englisch, fühlt er sich klar benachteiligt.

\section{„Hilf mir, es selbst zu tun!“ Maria Montessori}

Schulleiter*innen, Lehrpersonen, Sprachheilpädagog*innen, die Schulassistenz und alle an der Schule Tätigen, in welcher Kinder und Jugendliche im Fokus Hören beschult werden, sehen sich mit einer besonderen Aufgabe betraut. Sie sind es, die den Lernenden barrierefreies Partizipieren, Interagieren und autonomes Handeln ermöglichen sollen. Ist das geschafft, können auch Lernende im Fokus Hören all das, was hörende Kinder auch können, und werden zu einem gleichwertigen Mitglied innerhalb der Schulgemeinschaft. (Müller, 1996)

Es ist nicht möglich, diese*n eine*n Lernende* $n$ im Fokus Hören und ihre*seine Bedürfnisse nach Barrierefreiheit zu beschreiben, da jede* $r$ von innen individuell unterschiedliche Lernausgangslagen mitbringt. Dennoch kann davon ausgegangen werden, dass Kinder und Jugendliche im Fokus Hören, egal ob mit Hörgeräten beziehungsweise mit Cls versorgt oder auch ohne Hörbehelf, signifikante Defizite im Bereich der Aufmerksamkeit und Konzentration aufzeigen. Sie verzeichnen ebenso einen deutlich erhöhten Anstrengungs- und Ermüdungsgrad bei kognitiven Leistungen rund um die diversen Anforderungen im Unterricht oder in der Pause, im Vergleich zu ihren hörenden Mitschüler*innen. (Winkler, 2015) Daher bedürfen diese Kinder und Jugendlichen einer Begleitung und Unterstützung in Form von Kommunikationsstrategien und -tools sowie der Förderung ihrer sozial-emotionalen Kompetenz. (Gräfen, 2019)

\section{Identifikation der individuellen Lernausgangslage}

Möchte die Lehrperson mehr über die Lernenden im Fokus Hören erfahren, um diese in weiterer Folge angepasst unterstützen und begleiten zu können, erscheint es wichtig, sich ein umfassendes Bild zu machen. Mögliche Bereiche, die für die Erhebung der individuellen Lernausgangslage und der Bedürfnisse der Situation essenziell sein können, sind in der folgenden Abbildung angeführt, erheben jedoch keinen Anspruch auf Vollständigkeit: 


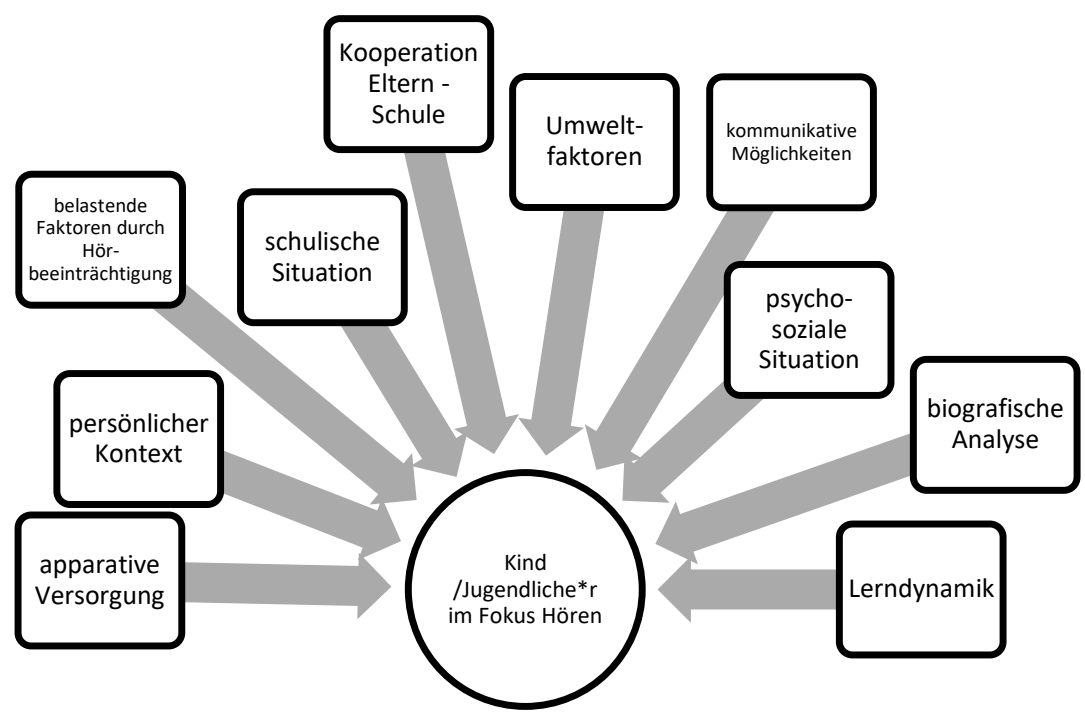

Abbildung 1: Identifikation individueller Lernausgangslagen | Eigene Darstellung

Alle genannten Faktoren gilt es im Sinne eines runden Tisches (Boban \& Hinz, 1998) mit möglichst vielen am und rund um das Kind Beteiligten, wie den Eltern, Therapeut*innen, wichtigen Bezugspersonen, Audiopädagog*innen, HNO-Fachärzt*innen, Pädakustiker*innen, und dem Kind selbst zu erheben und unter den unterschiedlichsten Blickwinkeln, zum Wohle der betroffenen Lernenden, zusammenzuführen.

\section{$\mathrm{PATH}^{2}$}

Ist die Erhebung der individuellen Lernausgangslage erfolgt, geht es darum, Teilhabeziele für die Lernenden zu entwerfen. Das Verfahren PATH - Planning Alternative Tomorrows with Hope (nach Pearpoint, O'Brien \& Forest, 1993) - bietet allen Beteiligten und dem betroffenen Kind selbst die Chance, gemeinsam Ziele und mögliche Veränderungen zusammenzubringen und zu entwickeln. Die Visualisierung (siehe Abb. 2) bietet zentrale Unterstützung und eine Strukturierungshilfe.

Die Erfahrung, einen gemeinsamen PATH für die und mit den Lernenden zu entwickeln, lässt alle Beteiligten die eigene Wahrnehmung mit jener der anderen abgleichen und im Perspektivenwechsel neue Blickwinkel sowie ein neues Verständnis entstehen. PATH hat sowohl eine klärende als auch eine orientierende Funktion und findet in vielen Zusammenhängen seinen Einsatz. (Wood, 2019) 


\begin{tabular}{|c|c|c|c|c|c|c|}
\hline Ist-Stand & Personen & Stärken & nächster Schritt & nāchster Monat & nächste Monate & \\
\hline $\begin{array}{l}\text { 2. SCHRITT } \\
\text { Wie sieht es } \\
\text { JETZT aus? } \\
\text { Gibt es } \\
\text { Typisches für die } \\
\text { gegenwärtige } \\
\text { Situation? } \\
\text { Autonomie, } \\
\text { Partizipation, } \\
\text { Kommuni- } \\
\text { kation, } \\
\text { Selbstbe- } \\
\text { stimmung, -. }\end{array}$ & $\begin{array}{l}\text { 3. SCHRITT } \\
\text { Wen oder was } \\
\text { wollen wir mit- } \\
\text { einbeziehen? } \\
\text { Welche } \\
\text { Unterstütz- } \\
\text { ungen, Helfer- } \\
\text { systeme, .. } \\
\text { haben wir? }\end{array}$ & $\begin{array}{l}\text { 4. SCHRITT } \\
\text { Was kann uns / } \\
\text { das Kind } \\
\text { stärken? } \\
\text {... auf schulischer } \\
\text { Ebene } \\
\text {... auf } \\
\text { individueller } \\
\text { Ebene } \\
\text {... auf gesell- } \\
\text { schaftlicher } \\
\text { Ebene } \\
\text {... }\end{array}$ & $\begin{array}{l}\text { 5. SCHRITT } \\
\text { Was tun wir } \\
\text { MORGEN? }\end{array}$ & $\begin{array}{l}\text { 6. SCHRITT } \\
\text { Rückblick nach } \\
\text { einem Monat... } \\
\text { Was ist bereits } \\
\text { getan worden? } \\
\text { Welche Ziele } \\
\text { sind geglückt? } \\
\text { Was muss noch } \\
\text { verändert } \\
\text { werden? }\end{array}$ & $\begin{array}{l}\text { 7. SCHRITT } \\
\text { In } 3 \text { bzw. } 6 \\
\text { Monaten ... } \\
\text { Was ist bereits } \\
\text { getan worden? } \\
\text { Welche } \\
\text { Einzelschritte } \\
\text { wurden gesetzt? } \\
\text { Wo muss } \\
\text { nachgebessert } \\
\text { werden? }\end{array}$ & $\begin{array}{l}\text { 1. SCHRITT } \\
\text { Wir reisen } \\
\text { mit der } \\
\text { Zeitmaschine } \\
\text { ein Jahr in } \\
\text { die Zukunft. } \\
\text { Welche } \\
\text { Erfolge } \\
\text { haben wir in } \\
\text { diesem Jahr } \\
\text { gefeiert? } \\
\text { Was wurde } \\
\text { geschaff? }\end{array}$ \\
\hline
\end{tabular}

Abbildung 2: PATH - Planning Alternative Tomorrows with Hope | Eigene Darstellung (nach Boban \& Hinz. 1998)

Um entlang des PATH-Verfahrens künftige Teilhabeziele möglichst konkret und wertungsfrei zu formulieren und Fördermaßnahmen sowie Unterstützungssysteme zu konzipieren, kann die ICF-CY ${ }^{3}$ (WHO, 2017) als Grundlage dienen.

\section{Bildung und Erziehung im Fokus Hören}

Damit nun autonomes Lernen und peerbezogene Kooperation für Lernende im Fokus Hören gelingen können, ist es wichtig, die organisatorischen und didaktischen Planungen an die individuellen Lernvoraussetzungen der Kinder und Jugendlichen anzupassen. (Leonhardt, 2019)

\section{Organisatorische Rahmenbedingungen}

Gute optische Bedingungen erleichtern das Lernen und Verfolgen des Unterrichtsgeschehens. Dazu tragen günstige Lichtverhältnisse (Raum- und Tafelbeleuchtung), die Vermeidung von Blendungen und stets aufrechter Blickkontakt zur Lehrperson zum Ablesen des Mundbilds bei. Die wichtigen akustischen Rahmenbedingungen werden durch Akustikplatten an der Decke des Klassenraumes, Vorhänge und Teppiche sowie Filzgleiter unter den Sesseln und Tischen optimiert. Der Einsatz von Übertragungsanlagen unterstützt Lernende bei der akustischen Wahrnehmung und dem Verstehen. Um die Klassenkolleg*innen für die Thematik der Hörbeeinträchtigung zu sensibilisieren, erscheint es wichtig, gemeinsam Verhaltensregeln aufzustellen, regelmäßigen sozial-strukturierten Austausch zu ermöglichen und kooperative Arbeits- und Spielmethoden anzubieten. (Fortmüller et al., 2016) 


\section{Didaktische Maßnahmen}

Um Lernende im Fokus Hören gelingend am Unterricht teilhaben zu lassen, muss keine andere Didaktik angewandt werden. Jedoch ist eine Bereitstellung optimaler Hör- und Absehbedingungen bei Unterrichtsgesprächen durch das Angebot von Gesprächsmitschriften über Overhead oder Smartboard, regelmäßige Sprech-, Hör- bzw. Absehpausen und Zusammenfassungen und Wiederholungen des Gesprochenen hilfreich. Die Anpassung der Lehrendensprache an das Niveau der Kinder und Jugendlichen, kurze Sätze, klare Fragen und eine deutliche Aussprache ohne Ironie oder Metaphern sowie eine Unterstützung dieser durch ÖGS ${ }^{4}$ und gezielte Impulse lassen die Lernenden besser verstehen und teilhaben. (Kramreiter, 2011)

Visualisierung, Strukturierung, Differenzierung und Individualisierung erleichtern Partizipation und Interaktion innerhalb und außerhalb des Unterrichts. Der Einsatz von Symbol- und Wortkarten unterstützt das Gesprochene. Klare, strukturierte Abläufe, Rituale und verständnissichernde Elemente schaffen Transparenz und Sicherheit für alle. Sowohl Scaffolding mit zusätzlichen Erklärungen zum Sprach- und Textverständnis, kollaboratives Arbeiten und individuell angepasstes Anschauungsmaterial als auch der häufige Wechsel der Sozialformen und Methoden erleichtern das Lernen für Kinder und Jugendliche im Fokus Hören. (Leonhardt et al., 2020)

Auch wir hören ... nicht alles. Nehmen wir uns trotzdem an den Ohren und hören wir hin, wenn uns Kinder und Jugendliche mitteilen wollen, wie sie am besten und barrierefrei lernen, partizipieren und interagieren können, um einen gemeinsamen PATH zu finden.

\section{Literaturverzeichnis}

Boban, I. \& Hinz, A. (1998). Diagnostik für Integrative Pädagogik. In H. Eberwein \& S. Knauer (Hrsg.), Handbuch Lernprozesse verstehen (S. 151-164). Weinheim: Beltz.

Flieger, P. (2020). Ermöglichen, nicht behindern. Zum Abbau von Barrieren für die Partizipation von Kindern mit Behinderungen in Schule und Unterricht. In S. Gerhartz-Reiter \& C. Reisenauer (Hrsg.), Partizipation und Schule, Perspektiven auf Teilhabe und Mitbestimmung von Kindern und Jugendlichen (S. 134-150). Wiesbaden: Springer.

Fortmüller, N., Hennies, J., Hintermair, M. \& Nedden, T. (2016). Kommunikative Partizipation und Integrationserleben hörgeschädigter Schülerinnen und Schüler an einer Schwerpunktschule. Empirische Sonderpädagogik 8, 2, S. 153-170.

Gräfen, C. (2019). Soziale Situation von Schülern mit Hörschädigung an der Allgemeinen Schule. Sprache-Stimme- Gehör, 43(03), S. 144-149.

Kramreiter, S. (2011). Integration von gehörlosen Kindern in der Grundschule mit Gebärdensprache und Lautsprache in Österreich. Wien: Universität Wien.

Leonhardt. A. \& Truckenbrodt, T.(2020). Schüler mit Hörschädigung im inklusiven Unterricht: Praxistipps für Lehrkräfte. München: Ernst Reinhardt.

Leonhardt, A. (2019). Grundwissen Hörgeschädigtenpädagogik. München: Ernst Reinhardt. 
Leonhardt, A. (2011). Inklusion als fachspezifische Aufgabe der Gehörlosen- und Schwerhörigenpädagogik. Sprache - Stimme - Gehör (35), S. $218 \mathrm{f}$.

Müller, R. J. (1996). ... ich höre - nicht alles! Hörgeschädigte Mädchen und Jungen in Regelschulen. Heidelberger sonderpädagogische Schriften, Bd. 21. Heidelberg: Edition Schindele.

Pearpoint, J., O'Brien, J. \& Forest, M. (1993) Planning Alternative Tomorrows with Hope. A Workbook for Planning Possible Positive Futures. Toronto.

WHO (2017). ICF-YC: Internationale Klassifikation der Funktionsfähigkeit, Behinderung und Gesundheit bei Kindern und Jugendlichen. Bern: Hogrefe.

Winkler, L.M. (2015). Psychische Begleiterscheinungen einer Hörbeeinträchtigung. Einfluss einer Hörbeeinträchtigung auf die subjektiv psychische Befindlichkeit und die Aufmerksamkeit nach kognitiven Aufgaben. Graz: Karl-Franzens-Universität.

Wood, H., O'Farrell, K., Bjerk-Andersen, C., Mullen, C. \& Kovshoff, H. (2019). The Impact of Planning Alternative Tomorrows with Hope (PATH) for Children and Young People. Educational Psychology in Practice, 35:3, pp. 326-338.

\section{Anmerkungen}

${ }^{1}$ Der Name des Schülers wurde aus datenschutzrechtlichen Gründen geändert.

2 PATH: Planning Alternative Tomorrows with Hope (Pearpoint, O’Brien \& Forest, 1993)

${ }^{3}$ ICF-CY: Internationale Klassifikation der Funktionsfähigkeit, Behinderung und Gesundheit bei Kindern und Jugendlichen (WHO, 2017)

4 ÖGS: Österreichische Gebärdensprache

\section{Autorin}

Manuela Buchegger-Pieber, MEd. BEd.

Seit 2019 Professorin an der Pädagogischen Hochschule Niederösterreich im Department für Diversität mit den Schwerpunkten Hören, ICF, Autismusspektrum, Peer-Mediation und Unterstützte Kommunikation, davor seit 1996 Volks- und Sonderschullehrerin an Schulen in Wien und Niederösterreich, Mitglied der Heilpädagogischen Gesellschaft NÖ und der Gesellschaft für Sensorische Integration Österreich.

Kontakt: ma.buchegger@ph-noe.ac.at 